\title{
Shunt infections in children: aetiology, treatment, cerebrospinal fluid concentration of intravenous antibiotics and therapeutic outcome in a I3-year material
} Kai Arnell*, Kristina Cesarini, Tomas Wester and Jan Sjölin

\author{
Address: Department of Paediatric Surgery, Neurosurgery and Infectious Disease, University Hospital, S- 75185 Uppsala, Sweden \\ Email: Kai Arnell* - kai.arnell@surgsci.uu.se \\ * Corresponding author
}

\author{
from 50th Annual Meeting of the Society for Research into Hydrocephalus and Spina Bifida \\ Cambridge, UK. 30 August - 2 September 2006 \\ Published: 2I December 2006 \\ Cerebrospinal Fluid Research 2006, 3(SuppI I):S38 doi:I0.II86/1743-8454-3-SI-S38
}

(C) 2006 Arnell et al; licensee BioMed Central Ltd.

\section{Background}

Despite improved operative technique and prophylactic antibiotics shunt infections still are a major problem in hydrocephalic children with risk for sequels and even mortality. The aim of the study was to analyse the incidence, aetiology, and therapeutic outcome of shunt-infections in paediatric hydrocephalus.

\section{Materials and methods}

All children (0-15 years) with hydrocephalus operated from January 1992 and December 2004 were retrospectively reviewed to identify shunt infections. Patients with shunt infections were further analysed focusing on aetiology, clinical presentation, glucose ratio, treatment, and outcome. In eight children the treatment was started with intravenous antibiotics alone and in these cerebrospinal fluid (CSF) antibiotic concentrations were monitored.

\section{Results}

During this period 472 shunt operations were performed on 237 children. 37 infections with positive culture from cerebrospinal fluid (33 cases) or from the valve ( 4 cases) were found. The infections occurred predominantly in children less than 1 year of age (57\%). In $80 \%$ of the cases skin bacteria, such as coagulase-negative staphylococci (18 cases), Staphylococcus aureus ( 7 cases) and Propionibacterium (5 cases) were found. Children less than 12 months of age were more commonly affected by systemic symptoms such as fever and redness over the shunt system than older children. Infections caused by S. aureus presented with fever and low glucose ratio in all the investigated cases. Propionibacterium infections were detected in 5 children, all presented with signs of distal obstruction. Only one of these had a pathologic glucose concentration. Infections secondary to blood stream infection occurred in 7 (19\%) children of all ages with systemic symptoms and high fever. Children with adequate systemic and intra-ventricular antibiotics recovered within 5 days. In children with intravenous antibiotics alone, cultures were still positive after 4-37 days and CSF antibiotic concentrations were low. Addition of intra-ventricular antibiotics resulted in recovery within a few days.

\section{Conclusion}

More than half of the infections were found in children less 12 months of age. Most frequently found bacteria were coagulase-negative staphylococci. Neonates and infants had more systemic symptoms than older children. Systemic antibiotics combined with intra-ventricular resulted in fast recovery and eradication of bacteria. 